\title{
Review
}

\section{Role and Impact of Cerebrolysin for Ischemic Stroke Care}

\author{
Dafin F. Mureșanu ${ }^{1,2}$, Livia Livinț Popa ${ }^{1,2}$, , Diana Chira ${ }^{1}$, Victor Dăbală ${ }^{1,2}$, Elian Hapca ${ }^{1,2}\left(\mathbb{D}\right.$, Irina Vlad ${ }^{1,2}$, \\ Vitalie Văcăraș ${ }^{1,2}$, Bogdan Ovidiu Popescu ${ }^{3}$, Răzvan Cherecheș ${ }^{4}$, Ștefan Strilciuc ${ }^{1,2} \mathbb{O}$ and Michael Brainin ${ }^{5}$
}

1 RoNeuro Institute for Neurological Research and Diagnostic, 400364 Cluj-Napoca, Romania; dafinm@ssnn.ro (D.F.M.); diana.chira@ssnn.ro (D.C.); victor.dabala95@gmail.com (V.D.); elianhapca@gmail.com (E.H.); irina.vlad001@gmail.com (I.V.); vvacaras@umfcluj.ro (V.V.); stefan.strilciuc@ssnn.ro (S.S.)

2 Department of Neuroscience, Iuliu Hatieganu University of Medicine and Pharmacy, 400083 Cluj-Napoca, Romania

3 Department of Neuroscience, Carol Davila University of Medicine and Pharmacy, 050474 Bucharest, Romania; bogdan_ovidiu_popescu@yahoo.com

4 Department of Public Health, Babes-Bolyai University, 400294 Cluj-Napoca, Romania; razvan.m.chereches@gmail.com

5 Department of Clinical Neurosciences and Preventive Medicine, Danube University Krems, 3500 Krems, Austria; michael.brainin@donau-uni.ac.at

* Correspondence: livia.popa@ssnn.ro

\section{check for}

updates

Citation: Mureșanu, D.F.; Livint Popa, L.; Chira, D.; Dăbală, V.; Hapca,

E.; Vlad, I.; Văcăraș, V.; Popescu, B.O.; Cherecheș, R.; Strilciuc, S,; et al. Role and Impact of Cerebrolysin for Ischemic Stroke Care. J. Clin. Med. 2022, 11, 1273. https://doi.org/ $10.3390 /$ jcm 11051273

Academic Editors: Antonio

J. Carcas-Sansuán and Alberto M. Borobia Pérez

Received: 13 January 2022

Accepted: 23 February 2022

Published: 25 February 2022

Publisher's Note: MDPI stays neutral with regard to jurisdictional claims in published maps and institutional affiliations.

Copyright: (C) 2022 by the authors. Licensee MDPI, Basel, Switzerland. This article is an open access article distributed under the terms and conditions of the Creative Commons Attribution (CC BY) license (https:// creativecommons.org/licenses/by/ $4.0 /)$.

\begin{abstract}
Stroke is still a significant health problem that affects millions of people worldwide, as it is the second-leading cause of death and the third-leading cause of disability. Many changes have occurred in the treatment of acute ischemic stroke. Although the innovative concepts of neuroprotection and neurorecovery have been vigorously investigated in a substantial number of clinical studies in the past, only a few trials managed to increase the number of promising outcomes with regard to the multidimensional construct of brain protection and rehabilitation. In terms of pharmacological therapies with proven benefits in the post-ischemic process, drugs with neurorestorative properties are thought to be effective in both the acute and chronic phases of stroke. One significant example is Cerebrolysin, a combination of amino acids and peptides that mimic the biological functions of neurotrophic factors, which has been shown to improve outcomes after ischemic stroke, while preserving a promising safety profile. The purpose of this paper is to offer an overview on the role and impact of Cerebrolysin for ischemic stroke care, by touching on various aspects, from its complex, multimodal and pleiotropic mechanism of action, to its efficacy and safety, as well as cost effectiveness.
\end{abstract}

Keywords: stroke; Cerebrolysin; early motor rehabilitation; ischaemic stroke; neurorehabilitation; neuroprotection; neurotrophic activity; neuroplasticity; thrombolysis; recovery; cerebrovascular disorders; brain diseases; neuroprotective agents; protective agents

\section{Introduction}

Stroke remains a serious health issue that impacts millions of individuals worldwide, representing the second-most common cause of mortality and the third-most common cause of disability [1]. Approximately $60-80 \%$ of all strokes are ischemic and result from thrombotic or embolic occlusion of a cerebral artery [2]. The management of acute ischemic stroke has undergone many changes. Regarding recanalization therapies such as thrombolysis and mechanical thrombectomy, the number of patients who may benefit from them is still low. Therefore, different therapeutic strategies have been developed, targeting the pathophysiological cascade that starts with ischemia and leads to irreversible tissue damage [3,4].

The innovative concepts of neuroprotection and neurorecovery have been actively researched in many clinical studies in the past. However, only a few trials in recent 
decades succeeded in increasing the number of positive results with reference to the broad concept of brain protection and rehabilitation, due to specific approaches that resulted in inconsistent evidence, therapeutic schemes that concentrated on suppressive strategies or the excessive research of the concept of monomodality (drugs that possess a single mechanism of action) [5].

Following an acute brain injury, there is an activation of an internal defensive array (known as the endogenous defense activity) consisting of two different pathways: neuroprotection (an immediate process, whose main target is the limitation of neuronal damage) and neurorecovery (which can be divided into three different branches-neurotrophicity, neuroplasticity and neurogenesis) [5].

The notion of multimodality, in neuropharmacological terms, refers to the binding of neuroprotection to the long-term reparatory processes that shape neuroregeneration, mirroring the physiological sequence of post-lesional endogenous regulation. Various attempts to integrate this concept were considered, with the use of monomodal drugs being ruled out as ineffective. Consequently, the multimodal agents with a pleiotropic neuroprotective effect in neurorehabilitation are considered a suitable solution based on current research [5].

Concerning the constellation of pharmacological therapies that could provide proven benefits in the post-ischemic process, the drugs with neurorestorative properties are effective in both the acute and chronic phases of stroke [6]. A significant advantage of neurorestorative drugs is their prolonged effectiveness, not being temporally restricted by pathologies such as brain ischemia. Because their availability exceeds the afferent therapeutic window of stroke (e.g., for tissue plasminogen activator-tPA), this type of pharmaceutical agent can be used for days, if not weeks, after an ischemic event. Restorative treatments should be coupled with rehabilitation, which likely acts synergistically to enhance neurological recovery [5]. Current data suggest that in over $70 \%$ of the stroke patients that benefit from thrombectomy and tPA, the perfusion rate of the affected tissues was still under the optimal parameters. Since complete tissue perfusion following such lesions is not feasible, many survivors develop further neurological deficits [6].

Cerebrolysin, a combination of amino acids and peptides that replicate biological effects of neurotrophic factors, is proven to exert beneficial outcomes when administered after ischemic stroke, while maintaining an encouraging safety profile. It reduces the number of procoagulant, prothrombotic and proinflammatory mediators, maintaining the normal function and health of the cerebral microvasculature after ischemia. An essential number of inflammatory cytokines is produced because of blood-brain barrier (BBB) injuries, caused not only by fibrin molecules, but also because of thrombolysis (with tPA). Therefore, the multimodal drug may enhance the therapeutic efficacy and safety of thrombolytic agents and thrombectomy, protecting the BBB [6].

As a result, future therapies may include neuroprotectants with more than one mechanism of action; therefore, multimodality should be systematically and intensively investigated, along with the discovery of novel agents and the thorough investigation of those that have demonstrated neuroprotective potential, not only before recanalization but also during the process. Subsequently, such actions could generate a further augmentation for the functional outcome, protecting the brain from both ischemia and reperfusion damage [6].

\section{Concepts of Neuroprotection and Neuroregeneration}

Stroke has immediate and long-term effects such as the impairment of movement, sensation, cognition, psychological and emotional functions, reducing independence and quality of life. Moreover, the neuroplastic changes that follow a cerebral infarction may occur over days, weeks, months or even years. However, despite all the progress in understanding the expansion of an ischemic event, the physiopathology of stroke is only partially known, the fundamental mechanisms of the brain that are related to its defense, protection and adaptation modulating processes are one of the enigmas and challenges of translational neurology [7]. 
Presently, the existence of an endogenous defense mechanism that incorporates neurobiological mechanisms, such as neuroprotection, neurotrophicity, neuroplasticity and neurogenesis, is already acknowledged. The central nervous system assesses controlling influence with modifying and repairing roles on different levels endogenously [8]. For this reason, their augmentation represents a therapeutic target [5].

The endogenous defense mechanism is activated following an acute injury such as an ischemic stroke through two anticorrelated mechanisms: the neuroprotection mechanism (with immediate action), and the (partially superposed) neuroregeneration mechanisms (neurotrophicity, neuroplasticity and neurogenesis) [9]. The two main anticorrelated processes aim in the first phase to reduce damage, leading to impairment, afterward aiming to repair the damage, followed by disability [9]. The fundamental biological processes and pathological mechanisms that comprise endogenous defense and damage mechanisms are summarized in Figure 1 [5].
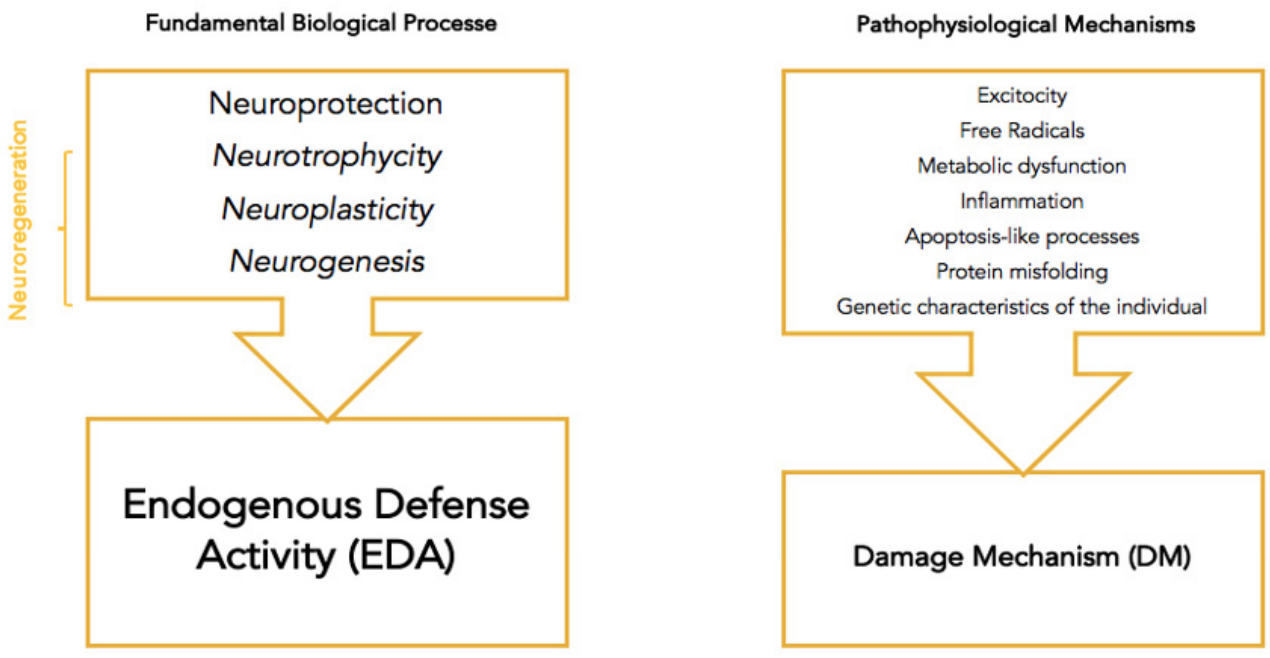

Figure 1. Endogenous defense activity (EDA) and damage mechanism (DM) [5].

Endogenous defense mechanisms consist of absolute and relative processes. Fundamental mechanisms lead to gene expression and protein synthesis, which play a restorative role. The other cell compounds, such as the cytoplasm, membrane or cytosol, are influenced by different (relative) mechanisms (e.g., ion channel blockers, agonists/antagonists of receptors, antioxidants, etc.), which determine neuroprotective activity expression $[1,5,10]$.

Pharmacological neuroprotection follows a similar organization. Neurotrophic factors and neurotrophic-like molecules control the absolute mechanisms, while ionic channel blockers influence the relative mechanisms, agonists and antagonists of certain receptors, antioxidants and other monomodal therapeutic agents $[1,5]$.

Neuroprotection, as part of the endogenous defense activity of the nervous system, is defined as the sum of all mechanisms allowing the neuron to functionally adapt against harmful factors. It is a short-term neurobiological process. The main aim of neurovascular protection is to preserve the components of the neurovascular unit, consisting of neurons, glial cells, endothelial cells, pericytes and matrix proteins. Any damage to parts of the neurovascular unit will lead to an apoptotic-like process, namely, anoikis $[5,8,10,11]$.

The nervous system can change and adjust its activity, functions, and interrelations, in association with structural modifications, in response to the damaging action of different (intrinsic and extrinsic) factors, this capacity being termed neuroplasticity. Neuroplasticity plays an important role in the recovery process after stroke because of its reorganizational capacity, covering not only the neuronal structure, but also the functional aspects and their interrelation $[1,10,12]$. It overlaps with neurotrophicity and neuroprotection, sharing common mechanisms [10]. Not all physiopathological processes and the connections between these two processes are known. There is, however, a certain degree of overlap 
between them [13]. All of these fundamental biological processes, as mentioned before, have an absolute aspect and a relative aspect that substantially contrast $[5,8]$.

Both excitotoxicity and inflammation possess a bivalent attribute: The role they have in developing an ischemic stroke is destructive and protective. While excitotoxicity occurs from the glutamate excess, and because of the consecutive surplus activation of the NMDA receptors, it leads, through proteolysis, to injury and cellular death, and in the case of a stroke, the inflammatory process expands through the activated immune cells. The two processes also share a common mechanism that is mediated through neurotrophic factors, which facilitate neuronal survival. Being part of neuroplasticity and neurotrophicity (for excitotoxicity), respectively (for inflammation), the endogenous actions of neuroprotection and neuroplasticity reveal the protective side of the whole activity (although certain conditions must be fulfilled, such as maintaining the proteolysis at an optimal level) $[5,8,14-19]$.

Finally, of the pathophysiological mechanisms (unlike apoptosis, which represents a normal process in the human body, with the role of maintaining and controlling cellular populations), apoptotic-like processes always produce negative effects, this being the reason for the need of counteraction through exogen and endogen mechanisms [5,8,20-22]. In conclusion, there is a need to concentrate on an attempt to develop pharmacological therapies that act in ways that can discontinue the apoptotic-like processes and turn the balance of the excitotoxicity and inflammation towards their positive effects, as opposed to the negative effects $[5,8,10,22]$.

The disability encountered after stroke varies greatly, from movement impairment to cognition and psycho-emotional status. Therefore, with the help of neurorehabilitation, there is an ongoing attempt related to amplifying the capacity of the neuroplasticity of the nervous system [11,22].

Neurogenesis represents the mechanism when new neurons are being produced from neural stem cells, and neurotrophicity describes the mechanism with which the cell constantly maintains its normal constitution and DNA expression $[5,8,23]$.

Neurotrophic factors, neurotrophic-like factors and genetic parameters are all processes that initiate the endogenous defense activity, as well as its governance $[5,10]$. They act on the absolute mechanisms, that lead to gene expression and protein synthesis, which both play a restorative role. Other cell compounds, such as the cytoplasm, the membrane or the cytosol are influenced by different (relative) mechanisms (i.e., ion channel blockers, agonists/antagonists of receptors, antioxidants, etc.), which determine neuroprotective activity expression $[5,10]$. Consequently, the need to activate and accentuate the endogenous systems (both internal and pharmacological) resides in the efficient counter of the pathophysiological mechanisms. This exogenous augmentation can be achieved using various interventions, from pharmacological to psychological ones. The use of molecules that mirror the structure and function of endogenous molecules has been proven to be beneficial, considering the complex continuous processes in which the latter are involved. For instance, it is known that the first $72 \mathrm{~h}$ after an ischemic event are of utter importance, considering the relevance that they have in the mechanisms of neuroprotection and neurogenesis and neuroplasticity $[5,10]$. Neurorehabilitation strives to enhance these mechanisms for improved outcomes $[5,7,8]$.

The physiopathological processes of stroke also occur in the stage between the vascular occlusion and cellular apoptosis, thus representing a target for pharmacological intervention, saving the neuronal population affected by the injury $[10,11,24]$. These defense mechanisms can be activated naturally or pharmacologically $[10,11]$.

In recent years, many attempts have been made to develop neuroprotective drugs targeting the pathophysiological cascade that starts with ischemia and leads to irreversible tissue damage. Despite inconsistent results from numerous clinical trials, some of them have been successful (Citicoline, Cerebrolysin, Erythropoietin, etc.). The typical pattern that all these molecules share is that they exhibit a pleiotropic mechanism against the ischemic cascade $[10,11,13]$. 
An important characteristic that an efficient neuroprotective drug should possess nowadays is multimodality, or the capacity to activate multiple biological mechanisms at the same time. The multimodal action is even more critical when the mechanisms of neuroplasticity and neurorecovery suffer from a long-term impairment, since many traditional pharmacological agents have an influence on a single mechanism [10]. The multimodal and pleiotropic effect provides the capacity to ensure, from a pharmacological perspective, the connection between the acute part, with a neuroprotection role, and the long-term part, with a regenerative role. The pharmacological consequences are summarized in Figure 2 [5].

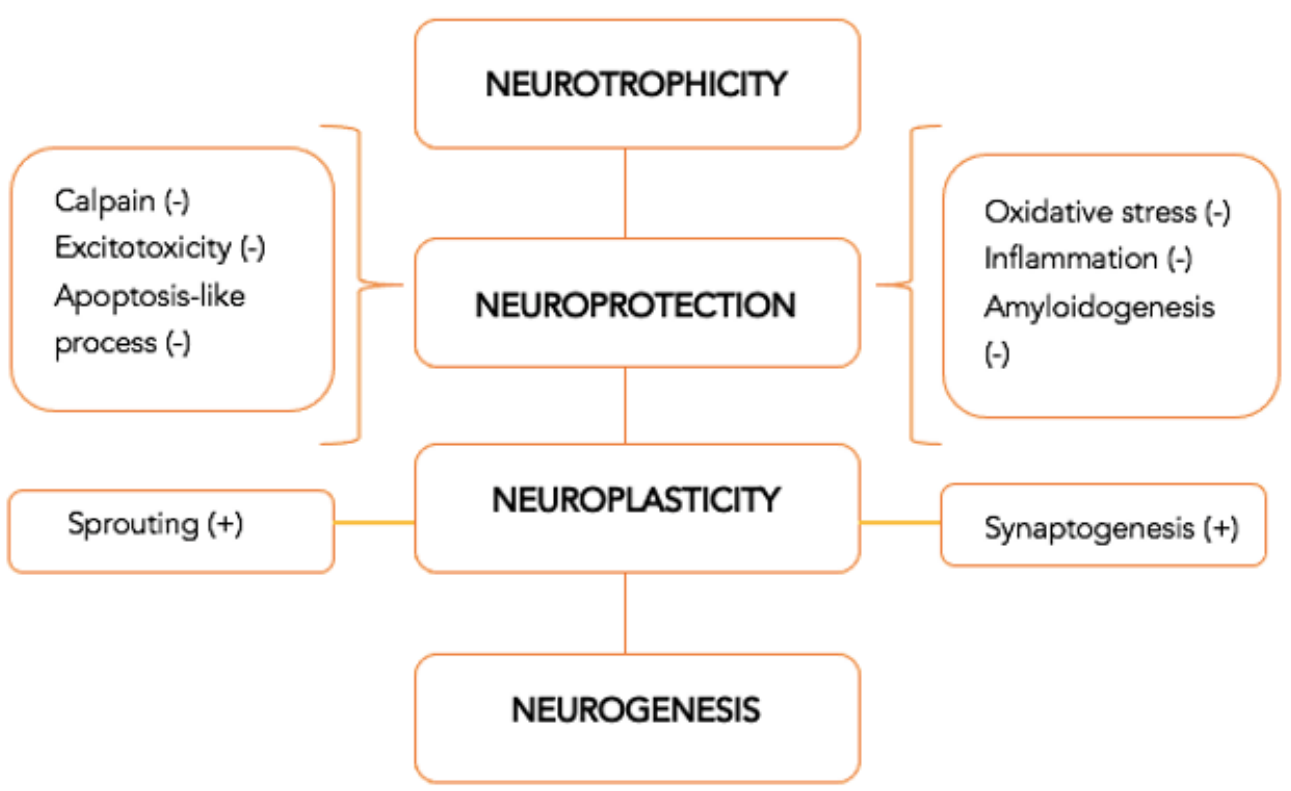

Figure 2. Multimodal drugs with pleiotropic neuroprotective effect-mechanism of action [5].

The ensemble of pathophysiological mechanisms denotes the importance of the multimodal action of the pharmacological therapeutic agents. For instance, glutamate has both an excitotoxic action in the first minutes and hours post-stroke, as well as a neuroregenerative action after a few hours [5,13]. The control of this change, vital for the protection of the brain, is assigned to the action of multimodal pharmacological agents, triggering the shift from neuroprotection to neuroplasticity $[5,13]$. This is one of the reasons why timing the expression of the implicated genes with the pharmaceutical effect of this type of drug has an exponential role in the pathogenesis of ischemia [9].

At present, the general therapeutic term (unimodal pleiotropic) refers to the usage of drugs that work through increasing the action of EDA exogenously, focusing only on neuroprotection $[5,9]$. Therefore, there is a need to concentrate on the pharmacological performance and neuroprotective effects of the drugs with multimodal and pleiotropic activity, (i.e., the biological agents), as opposed to the unimodal acting ones, since the results that derived from the latter category are not consistent enough [5]. Based on this principle, Cerebrolysin is an example of an agent with a complex action mechanism. It possesses a proven multimodal, pleiotropic action, which provides not only immediate neuroprotection, but also long-term neuroregeneration, by activating endogenous responses that can be observed in a certain number of cerebrovascular and neurodegenerative diseases, including stroke [9].

Cerebrolysin is a complex compound, which is composed of peptides with active, neurotrophic activity and modulation, and free amino acids, which, through its multimodal action, promotes neurotrophic stimulation (through the survival and maintenance of the phenotype of highly differentiated cells), modulates neuroprotection against noxious agents (facilitating changes in the plasticity of neurons and synapses), and benefits the neuronal 
metabolism through an increase in resistance to hypoxic conditions and prevention of lactate accumulation (lactic acidosis) $[9,25,26]$.

This multimodal action of Cerebrolysin was shown in animal and in vitro studies, consisting of a reduction in programmed cell death and free radicals' development, the regulation of the inflammatory response, and a reduction in the toxic actions of neurotransmitters (excitotoxicity), all leading to neuroprotection; a reduction in regulating the increase in the number of synapses, leading to neuroplasticity; and a reduction in neurovascular reconstruction, leading to neurogenesis in the dentate gyrus [27-33].

This pharmaceutical agent increases neurogenesis and oligodendrogenesis, by activating the Sonic Hedgehog pathway, with a role in the evolution and structuring of the brain. One example is the Gli complex, which increases neurorecovery [34]. The capacity of Cerebrolysin to induce neurorecovery, along with the standard treatment of stroke, is the reason why this potent drug represents an efficient option as an add-on treatment for stroke rehabilitation [9]. It was suggested that Cerebrolysin has a more accentuated effect on neuroregeneration (neuroplasticity and neurorestoration) than on neuroprotection [13].

\section{Clinical Trials}

\subsection{Efficacy of Cerebrolysin}

Cerebrolysin has been the subject of multiple clinical trials, the majority of which have yielded encouraging results in terms of multimodal and pleiotropic activity. Various studies have shown that the intravenous administration of Cerebrolysin can improve the neurological outcomes of patients who have had an acute ischemic stroke, as well as its beneficial association with other pharmaceuticals and types of physical, occupational or speech therapies. Tran et al. studied the effect of Cerebrolysin with nootropics in the treatment of acute ischemic stroke (AIS) patients. Their results showed that Cerebrolysin, alone or in combination with other such pharmaceutical agents, was found to be safe and beneficial in the treatment of acute ischemia, in both the acute and recovery stages, indicating that it should be used in everyday clinical practice [35]. Chang et al. focused on the combination of Cerebrolysin with standardized rehabilitation therapy. The results showed that in individuals with severe motor impairment caused by acute ischemic stroke, conventional rehabilitation therapy combined with Cerebrolysin delivers extra benefits to conventional rehabilitation therapy alone in motor recovery [36]. An earlier study by Chang et al. (which used for the first-time neuroimaging for motor network plasticity evaluation when administering Cerebrolysin) revealed a positive influence of Cerebrolysin on cerebral tissue related to motor function, but no significant difference was found between the two groups [37]. Xue et al. conducted a clinical trial to test and assess the efficacy and safety of DL-3-n-butylphthalide (NBP) and Cerebrolysin in minimizing neurological and behavioral impairment after acute ischemic stroke. The findings of this study suggested that a 10-day treatment with NBP or Cerebrolysin could be used safely and may have favorable benefits in patients with AIS, especially in mild cases. However, NBP appeared to be better than Cerebrolysin at improving the short-term prognosis of acute ischemic stroke [38].

The study of Muresanu et al., CARS 1, showed a positive influence of Cerebrolysin on both functional and overall outcomes in early stroke rehabilitation. More precisely, participants who received the drug had better upper-extremity motor function at 90 days, than patients who received placebo [4]. Following CARS 1, a study of Guekht et al., CARS 2 aimed follow the design of the first trial, on a larger scale. However, this study did not support the findings of CARS 1, but Cerebrolysin was tolerated in its sample [39]. Razei et al. concentrated on just Cerebrolysin, aiming to see how it affected neurological results and the brain blood flow. The results showed that Cerebrolysin might help patients with acute focal ischemic stroke improve their neurological results, also affecting the pulsatility index (PI) of the middle cerebral artery [40]. Lang et al. conducted another trial to see if combining alteplase (rt-PA) with Cerebrolysin was safe and effective in reducing impairment following an acute ischemic stroke. They concluded that the neurotrophic 
agent combined with rt-PA was safe for the treatment of AIS, although it did not improve prognosis at 90 days. However, compared to the placebo group, considerably more patients had a favorable response in neurological outcome measures during the 10-day therapy period with Cerebrolysin [41]. Stan et al. assessed the efficiency of Cerebrolysin combined with post-stroke early rehabilitation, showing positive results in the cohort treated with Cerebrolysin. The authors reported improved overall neurological health and reduced impairment for the patients treated with Cerebrolysin. There were $28.5 \%$ more independent patients in the intervention group than the control group, showing that this study's positive findings could be effectively used in contemporary clinical practice [42]. Heiss et al. aimed to see whether the treatment with Cerebrolysin was suitable and safe in patients with acute ischemic stroke. The validating endpoint in this trial revealed no differences between the treatment groups. However, a favorable outcome trend was observed in the heavily impacted patients treated with Cerebrolysin [43]. Additional information regarding the studies we have referred to may be found in Table 1.

\subsection{Safety Profile of Cerebrolysin}

The safety of the neurotrophic drug Cerebrolysin was previously evaluated in various studies, presenting heterogeneous results regarding demographics, time of inclusion, administered dose and time of assessment/follow-up.

A trial published in 2012 reported a lower drop-out of patients from the study, due to side effects in the Cerebrolysin group, versus in the placebo group, concluding that there were no significant differences between the two categories. The comparison of the two clusters (Cerebrolysin and placebo) revealed no significant differences regarding side effects, severe side effects, and mortality. Additionally, no significant changes in vital parameters or laboratory test results were observed in patients treated with Cerebrolysin in the acute phase of ischemic stroke. It is noteworthy that during the analysis of the subcategory of patients with a NIHSS $>12$ points, a lower death rate could be associated with the intervention group, compared to the placebo group [43].

Another randomized clinical trial that involved Cerebrolysin mentioned a low percentage (under $5 \%$ ) of patients from the intervention group that were forced to leave the study due to adverse events. Of these, only two patients dropped out of the study prematurely due to adverse reactions. No deaths were reported in patients that received the neurotrophic treatment. Regarding adverse reactions, $2.9 \%$ of patients in this group and $6.7 \%$ of those receiving a placebo had a major side event with a complete resolution, considered to be unrelated to the administered agent. In the Cerebrolysin-treated group, severe side effects were mainly represented by acute myocardial infarction, severe peripheral ischemia, and renal colic, all of which underwent resolution during this study. According to the study protocol, the prolonged hospitalization of these patients, in addition to early rehabilitation, could be an explanation for the low rate of procedures regarding major secondary events. Additionally, relevant for this case was the absence of significant changes in the Cerebrolysin-treated group compared to the placebo-treated group, regarding vital and laboratory parameters [4]. 
Table 1. Trials involving the effect of Cerebrolysin on acute ischemic stroke in the past 9 years.

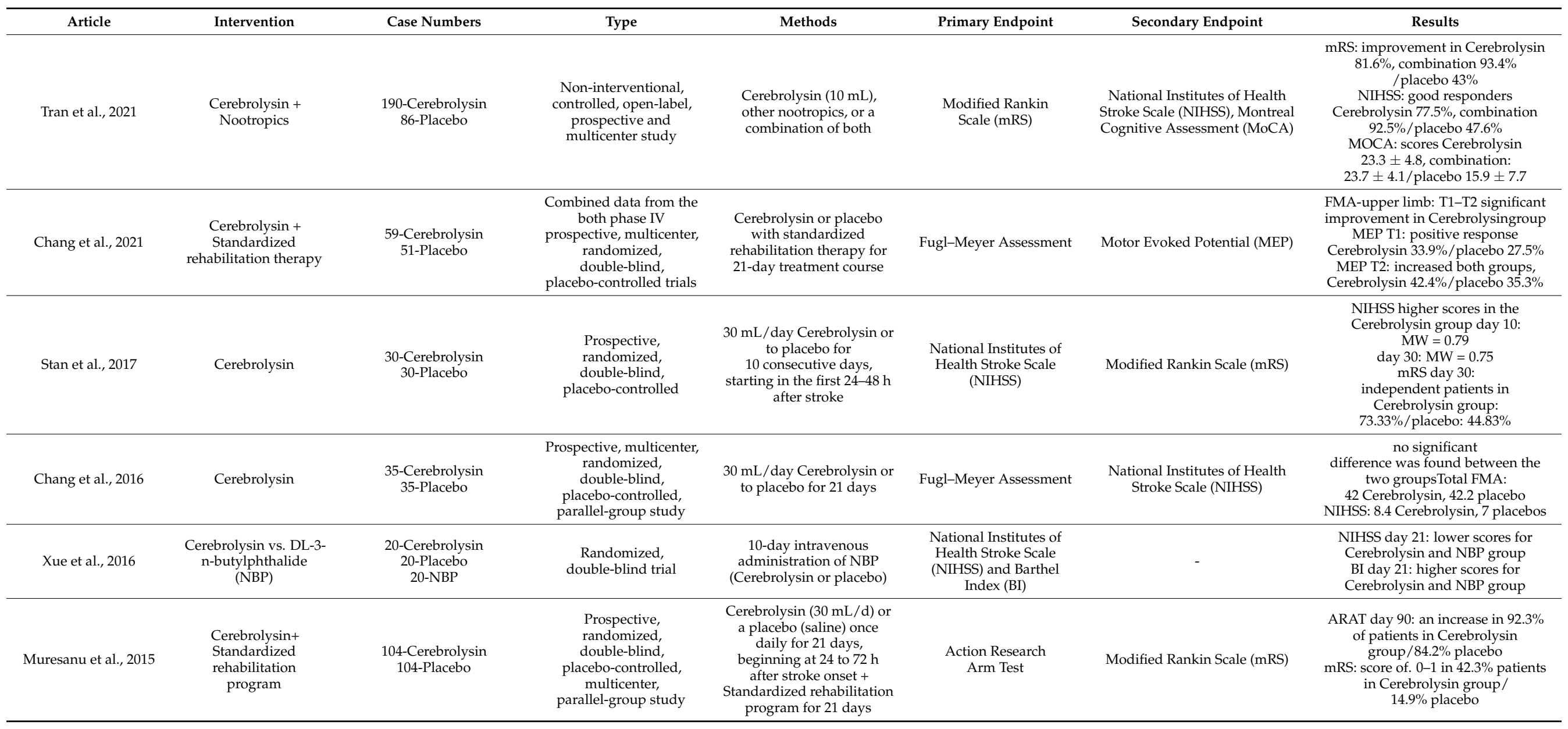


Table 1. Cont.

\begin{tabular}{|c|c|c|c|c|c|c|c|}
\hline Article & Intervention & Case Numbers & Type & Methods & Primary Endpoint & Secondary Endpoint & Results \\
\hline Guekht et al., 2015 & Cerebrolysin & $\begin{array}{l}\text { 120-Cerebrolysin } \\
\text { 120-Placebo }\end{array}$ & $\begin{array}{c}\text { Prospective, } \\
\text { randomized, } \\
\text { double-blind, } \\
\text { placebo-controlled, } \\
\text { multicenter, } \\
\text { parallel-group study }\end{array}$ & $\begin{array}{l}\text { Cerebrolysin }(30 \mathrm{~mL} / \mathrm{d}) \text { or } \\
\text { a placebo (saline) }\end{array}$ & $\begin{array}{l}\text { Action Research } \\
\text { Arm Test }\end{array}$ & $\begin{array}{l}\text { Gait velocity, fine motor } \\
\text { function, global neurological } \\
\text { status, disability, quality of } \\
\text { life, neglect }\end{array}$ & $\begin{array}{l}\text { No end points showed significant } \\
\text { improvement at } 90 \text { days for } \\
\text { Cerebrolysin group } \\
\text { mild baseline levels of impairment } \\
\text { showed improvement after } 90 \text { days } \\
\text { in placebo group }\end{array}$ \\
\hline Razei et al., 2014 & Cerebrolysin & $\begin{array}{l}\text { 23-Cerebrolysin } \\
\text { 23-Placebo }\end{array}$ & $\begin{array}{c}\text { Randomized, } \\
\text { double-blinded, } \\
\text { placebo-controlled trial }\end{array}$ & $\begin{array}{l}\text { Cerebrolysin }(30 \mathrm{~mL}) \\
\text { diluted in normal saline } \\
\text { daily or Normal saline } \\
\text { alone, adjunct to } 100 \mathrm{mg} \text { of } \\
\text { aspirin daily for } 10 \text { days }\end{array}$ & $\begin{array}{l}\text { National Institutes of } \\
\text { Health Stroke } \\
\text { Scale (NIHSS) }\end{array}$ & $\begin{array}{l}\text { Mean flow velocity and PI of } \\
\text { cerebral arteries }\end{array}$ & $\begin{array}{c}\text { NIHSS day } 60 \text { and } 90: \text { lower values } \\
\text { in the Cerebrolysin group } \\
\text { mean flow velocity day } 30: \text { higher in } \\
\text { the placebo group median }=53, \\
\text { Cerebrolysin median }=45 P \mathrm{PI} \text { lower in } \\
\text { the Cerebrolysin group } \\
0.85 / \text { placebo } 1.1\end{array}$ \\
\hline Lang et al. 2013 & $\begin{array}{l}\text { Cerebrolysin }+ \\
\text { Alteplase }\end{array}$ & $\begin{array}{l}\text { 60-Cerebrolysin } \\
\text { 59-Placebo }\end{array}$ & $\begin{array}{l}\text { Placebo-controlled, } \\
\text { double-blind trial }\end{array}$ & $\begin{array}{l}\text { Cerebrolysin }(30 \mathrm{~mL}) \text { or } \\
\text { placebo }(1 \mathrm{~h} \text { after } \\
\text { thrombolytic treatment) } \\
\text { starting within three-hours } \\
\text { after onset of symptoms, } \\
\text { given for } \\
10 \text { consecutive days }\end{array}$ & $\begin{array}{l}\text { Modified Rankin } \\
\text { Scale (mRS) }\end{array}$ & $\begin{array}{c}\text { National Institutes of Health } \\
\text { Stroke Scale (NIHSS), Glasgow } \\
\text { Outcome Scale (GOS), Barthel } \\
\text { Index (BI) }\end{array}$ & $\begin{array}{l}\text { mRS day 90: no significant } \\
\text { improvement in Cerebrolysin group } \\
\text { vs. placebo } \\
\text { NIHSS, GOS, BI: no significant } \\
\text { improvement in Cerebrolysin group } \\
\text { vs. placebo }\end{array}$ \\
\hline Heiss et al., 2012 & Cerebrolysin & $\begin{array}{l}\text { 529-Cerebrolysin } \\
\text { 541-Placebo }\end{array}$ & $\begin{array}{c}\text { Double-blind, } \\
\text { placebo-controlled } \\
\text { randomized clinical trial }\end{array}$ & $\begin{array}{l}30 \mathrm{~mL} \text { Cerebrolysin daily } \\
\text { or placebo (saline solution) } \\
\text { given as intravenous } \\
\text { infusion for } 10 \text { days in } \\
\text { addition to aspirin } \\
\text { (100 mg daily) }\end{array}$ & $\begin{array}{l}\text { Modified Rankin Scale } \\
\text { (mRS), National } \\
\text { Institutes of Health } \\
\text { Stroke Scale (NIHSS), } \\
\text { Barthel Index (BI) }\end{array}$ & $\begin{array}{c}\text { Responder analysis and } \\
\text { global test }\end{array}$ & $\begin{array}{l}\text { NIHSS day } 90: \text { improved by } \\
6 \text { Cerebrolysin } / 5 \text { placebo } \\
\text { BI: } 30 \text { for both groups } \\
\text { mRS: } 2 \text { for both groups } \\
\text { global test MW }=0.50 \\
\text { CI }=0.46\end{array}$ \\
\hline
\end{tabular}


A phase IV clinical trial, which included patients with moderate or severe motor dysfunction after a stroke, showed similar results between the intervention and placebo groups concerning the vital signs and laboratory values, while each group reported a single serious adverse event, the one recorded in the Cerebrolysin group being an episode of cholecystitis caused by gallstones, which remitted after some time [37].

Concerning the use of Cerebrolysin in patients who received intravenous thrombolytic treatment with Alteplase (rt-PA), no general increase in the frequency of deaths, severe side effects or adverse effects were reported, compared to the control group. In contrast to the other clinical trials, it is worth mentioning the differences in the timing of the administration of rt-PA, the shorter time between stroke-onset to rt-PA perfusion (onset to needle time), as well as the time of administration of Cerebrolysin between the placebo-group and the intervention group and the side effects (i.e., brain hemorrhage) that were attributed to the Alteplase. In this case, there were also no changes in vital or laboratory parameters related to the use of the neurotrophic drug [41].

A meta-analysis of nine randomized clinical trials reported a lower death rate in the Cerebrolysin group compared to the placebo group. When it comes to the calculated OR, it showed slightly lower results in the placebo group, but was statistically not significant. Moreover, the percentage of patients who reported severe side effects, and the one patient who reported at least one side effect, were comparable between the two groups. It is worth mentioning that only seven out of eight studies had available data regarding serious adverse events and adverse events [44].

Another meta-analysis focusing on the results of two corresponding stroke trials (CARS-1 and CARS-2 - both presented in Table 1) assessed the tolerability and safety of Cerebrolysin on motor function recovery in patients after stroke. It was observed that the number of patients treated with Cerebrolysin that reported at least one adverse event (AE) was similar to the events of the patients in the placebo group. Moreover, most AEs were rated as mild in terms of severity. Regarding serious adverse events (SAEs), fewer than 5\% of patients suffered from SAEs in each group and none of them was reported to be related to study medication [45]. In general, most of the adverse reactions reported in studies among patients who received Cerebrolysin were transient and could not be correlated with medication administration [13].

A 2021 systematic review and meta-analysis assessed the safety of administrating Cerebrolysin after acute ischemic stroke compared to a placebo. Based on the analysis of 12 randomized controlled trials, the safety profile of Cerebrolysin was assessed using not only the 12 RCT study population (2202 patients) but also subgroups. Considering the SAEs, death, AEs, and NF-SAEs of Cerebrolysin compared to a placebo, Cerebrolysin showed a good safety profile. Moreover, a trend toward SAE reduction was observed in the Cerebrolysin subgroup of patients with moderate-to-severe stroke, compared to the placebo [46].

Lastly, another systematic review assessed the benefits of Cerebrolysin in the treatment of acute ischemic stroke and the potential risks that the agent could possess during therapy. The results of the review concluded that Cerebrolysin made little to no difference regarding the risk of death from any cause after an ischemic stroke, the total number of subjects with SAE, the number of SAEs that resulted in death and the total number of patients who had any less SAEs. Referring to the combination of Cerebrolysin and standard therapy, the review reported an increase in the number of patients who had SAEs that did not result in death, compared to the groups who were assigned to standard therapy (alone or with placebo) (4 studies-1435 patients). Finally, the review did not find sufficient evidence regarding the risk of mortality and the need of continuous care at the end of the admitted studies [47].

All things considered, the safety of Cerebrolysin was studied in several different trials. There were no major differences registered compared to the placebo in terms of adverse effects (serious or fatal). Furthermore, there were no significant changes in vital and laboratory parameters. 


\section{Observational Research-Effectiveness Studies}

Often, an intervention is analyzed in terms of efficacy and effectiveness by clinicians and policymakers. One of the differences between these concepts is that efficacy trials (explanatory trials) play a role in determining whether the intervention produces the expected result, under ideal conditions. On the other hand, effectiveness trials (pragmatic trials) have the role of quantifying the degree of the beneficial effect in the context of "real world" circumstances. As a result, it is important to acknowledge that study designs and effectiveness trial hypotheses are developed by taking into consideration routine clinical practice and outcomes from interest in clinical decisions [48]. An extensive literature research, using Pubmed, was conducted with a focus on observational studies of the effectiveness of Cerebrolysin in stroke. Very few observational studies were identified; the most significant ones are presented below.

A retrospective study on the effectiveness of Cerebrolysin in post-stroke spasticity, was conducted on 50 patients ( 23 in the Cerebrolysin group and 27 in the control group). They received Cerebrolysin for 30 days, with $10 \mathrm{~mL} /$ day administered intramuscularly. Additionally, both groups participated for a minimum of 2 times a week in standardized physical and occupational rehabilitation therapy. Efficacy at day 30 was assessed with modified Ashworth scale (MAS), manual muscle testing (MMT) and modified Rankin scale. Cerebrolysin proved safe and effective; it showed a significant improvement in limb spasticity compared to the control group. In the Cerebrolysin group, strength and global function were also improved [49].

In a study by Kim et al., the effectiveness of Cerebrolysin on the state of consciousness was assessed in stroke patients with a minimally conscious state (MCS) in an observational retrospective study. Seventy-five patients with ischemic and/or hemorrhagic stroke with MCS according to the Coma Recovery Scale-Revised (CRS-R) were included during a period of 3 years. The Cerebrolysin group consisted of patients that had received Cerebrolysin $10 \mathrm{~mL}$ iv for a minimum of 20 days; the control group were patients with no Cerebrolysin administration. In addition, they received rehabilitation-physical and occupational therapy. Patients were assessed at discharge ( $\sim 2$ months) according to the Coma Recovery Scale-Revised (CRS-R) and, after eliminating confounders, showed a significant improvement, especially in the Oromotor and Arousal subscales. Furthermore, no safety issues were identified [50]. Based on our queries, the most significant studies identified in the literature research were interventional.

\section{Cost-Effectiveness Research}

Despite its high physical and cognitive burden, stroke also causes a tremendous economic burden worldwide. Stroke accounts for around 3 to 4 percent of the overall healthcare costs in Western countries [51]. Based on a recent systematic review by Rochman et al., it was observed that direct medical costs accounted for 86.2 percent and 13.8 percent of the overall cost [52]. These costs include medication, which plays an enormous part in treating stroke.

Cost-effectiveness analyses (CEAs) are reliable tools for health system stakeholders (both policy and decision makers) assessing the value (characterized by costs and outcomes within a predefined time-point) of pharmacological therapies [53,54]. CEA is typically accompanied by a budget impact analysis (BIA), which evaluates how affordable a pharmacological intervention is in a system with limited resources, before approval or reimbursement processes. Given the high competition for limited financial means within each country and the particularities of national insurance systems, CEAs and BIAs are recommended to be conducted for each alternative intervention [54]. Few economic evaluations have assessed how cost-effective the pharmacological therapy with Cerebrolysin is or its impact on national health budgets.

Kulikov and Abdrashitova et al. aimed to evaluate the cost effectiveness of Cerebrolysin against standard therapy for patients diagnosed with moderate or severe stroke. Based on the reported results, Cerebrolysin was the dominant therapy, as cost-effectiveness 
ratios (CER) were lower (EUR 6920) than standard therapy (EUR 9287) [55]. Kulikov and Abdrashitova et al. have also been developing a complementary budget impact analysis (BIA) since 2015, which showed that Cerebrolysin has lower direct costs associated with medical care (inpatient and outpatient care, pharmacotherapy, emergency care and neurorehabilitation) and indirect costs (loss of productivity caused by sick leave, disability, and death). The costs incurred by stroke patients included in the Cerebrolysin group were reduced by EUR 1314 compared to the cost incurred by patients receiving standard therapy (EUR 7552) [56]. Walter et al. showed that, in patients diagnosed with acute ischemic hemispheric stroke and treated with a combination of alteplase and Cerebrolysin, lower costs were reported (EUR 61,468.67) compared with patients receiving only alteplase. The treatment strategy involving neuroprotective agents had reduced costs attributable to acute ischemic CVA care and nursing homes [57].

While existing studies are scarce, there is a signal that Cerebrolysin is probably costeffective, with the potential to reduce the economic burden on national budgets, both as standard treatments in patients with different levels of severity $[55,56]$ or in combination with another pharmacological therapy (i.e., alteplase) [57] in countries with different types of health insurance [58,59].

\section{Cerebrolysin Recommendations in Guidelines}

Cerebrolysin has been featured in different guidelines regarding its administration in the acute phase of stroke, as well as during the chronic phase, for its evidence-based role in rehabilitation [46]. In 2020, Cerebrolysin was recommended in the Stroke Rehabilitation Clinician Handbook, to be administered in the rehabilitation process of the hemiplegic upper limb, stating the possibility of improvement of motor function, agility, and selfreliance during daily activities using Cerebrolysin. The recommendation was based on randomized controlled trials, with Cerebrolysin being administered in $30 \mathrm{~mL}$ dosage on $70 \mathrm{~mL}$ saline, once daily, intravenously for three weeks combined with physical or occupational therapy, or the same dosage and administration route as before, but for six weeks combined with standard rehabilitative therapy [60].

Cerebrolysin was also recommended as part of the pharmacological rehabilitation of the motor-deficient upper limb after stroke in the 2020 revised guideline of the German Society of Neurorehabilitation regarding the rehabilitation of the paretic upper limb. The recommendation was evidence- and consensus-based, with the group in charge of the guideline's conceptualization, The German Society for Neurorehabilitation. The guideline recommends the administration of Cerebrolysin in the acute and subacute phase of stroke in patients with a pertinent upper limb motor deficit, ideally 24 to $72 \mathrm{~h}$ after stroke, intravenously, daily, for 21 days (if tolerated). The aim of using Cerebrolysin is to enhance upper limb motor capacity and overall functional improvement, together with rehabilitation. Currently, Cerebrolysin is approved in Austria [61].

The 2021 European Academy of Neurology and European Federation of Neurorehabilitation Societies guideline on pharmacological support in early motor rehabilitation after acute ischaemic stroke recommends using two pharmacological agents, namely, Cerebrolysin and Citalopram. Cerebrolysin is recommended as a pharmacological add-on to the early motor rehabilitation in acute stroke with a dose of $30 \mathrm{~mL} /$ day administered intravenously, for a minimum of 10 days [62]. The existing evidence regarding the role and efficacy of Cerebrolysin in acute stroke was assessed and recommendations were made, using the Grading of Recommendations Assessment, Development and Evaluation (GRADE) framework, by the task force comprised by representatives from both the European Academy of Neurology (EAN) and the European Federation of Neurorehabilitation (EFNR) and from six European countries [62]. Six meta-analytics pathways (PICOs) were applied for scale classification: with the primary and secondary being the early motor performance at 30 days and at 90 days. Neurological function at 30 and 90 days and global functional outcome at 30 and 90 days, respectively, were considered for the safety profile when making the recommendations. Data extraction was followed by a meta-analysis [62]. 
In the AHA/ASA 2019 Guidelines for the Early Management of Patients with Acute Ischemic Stroke (2019 Update to the 2018 Guideline), aspects of neuroprotective therapy are briefly stated in the "General Supportive Care and Emergency Treatment" section. Although an A-Level of Evidence (LOE) was associated with the administration of neuroprotective agents, no beneficial effect regarding the patient's status was considered, therefore falling in Class of Recommendation (COR) III. Therefore, the guideline does not recommend the administration of any medicative and non-medicative agents with an assumed neuroprotective activity in the acute phase of ischemic stroke. Nevertheless, no randomized controlled trial (RCT) of Cerebrolysin was specifically included in the assessment process, which encompassed high-dose albumin and magnesium infusion only [63]. A similar approach to recommendation for post-stroke pharmacological intervention to aid neurorehabilitation is present in the 2016 AHA/ASA guidelines on Stroke Rehabilitation and Recovery [64].

\section{Conclusions}

Given the high frequency and poor prognosis of acute ischemic stroke, it is vital to discover effective medicines to help people with the disease enhance their neurological and cognitive skills. Based on the complex pathophysiological cascade associated with brain ischemia, a multimodal approach, targeting various critical mechanisms, appears to be a key future approach to enhance therapy. Agents that exert an endogenous activity should be considered the essential elements for the effective development of therapies that target the protection and recovery of the brain from an acute CNS lesion. These agents have the potential to act at the DNA level, with the activation of synergic molecular mechanisms that provide the reintegration of homeostasis of the damaged neurological tissues [5]. The multimodal and pleiotropic action of Cerebrolysin leads to immediate neuroprotection and long-term regeneration [9]. An essential advantage of this neuroprotective drug is the argument that it can be widely used without relevant restrictions. Furthermore, there is no time window limitation for the drug administration, showing a high safety profile and being well-tolerated. Equally important, there is a considerable body of evidence that Cerebrolysin protects the brain against the impact of the ischemic cascade and supports the whole neuronal reorganization process $[10,13]$.

Brain plasticity is the most important process of a synaptic phenomenon that is mainly a stimulus-dependent process. Recovery after stroke is a time-consuming and complex process, in which specific large brain lesions require not only new anatomical substrates, but also the renewing or creation of new network connections. It is important to understand that stroke is not only a regional impairment of the infarcted area, but its involvement in the distribution of whole-brain networks results in a broad spectrum of dysfunction and disability [5]. At the same time, for the functional outcome after stroke, acute rehabilitation, emphasizing timing and intensity, is of utmost importance. While early mobilization after stroke is recommended in clinical guidelines, it remains controversial, since it can also cause harm when the intensity is too high [64].

Neurological disorders represent a major burden globally, with regional and worldwide variability. Unfortunately, regardless of region, stroke predominates, especially when taking DALY (disability-adjusted life year) into account. The aim of the medical intervention in stroke, ranging from primary prevention to acute treatment, hospitalization, secondary prevention, and rehabilitation, is to lessen stroke occurrence, disability, and mortality [62]. The crucial role of proper early motor rehabilitation to reduce the debilitating consequences of stroke could be assisted by pharmacological support [13,60-62]. Further work is required, especially on understanding the balance between excitatory and inhibitory signals, determining the effects that injury has on them and, of course, identifying those targets that can efficiently control and maintain the equilibrium. Such a level of information would facilitate an accurate modulation of the neuronal network, leading to the activation of a localized mechanism of plasticity, while preserving stability in other areas [65]. 
Cerebrolysin is already contributing to the pharmacological armamentarium that clinicians have at their disposal in many countries worldwide $[9,10,13]$. Despite the prolific literature that has been published on the efficacy and safety of the add-on intervention for post-stroke neurorehabilitation, some limitations of clinical studies (e.g., small sample sizes) warrant additional investigation via large, high-quality confirmatory trials, as well as clinical and cost-effectiveness studies [4,35-43]. Improving this body of evidence in the future will impact current clinical guideline recommendations for Cerebrolysin after ischemic stroke, likely clarifying divergent opinions on this topic [60-62].

Author Contributions: Conceptualization: D.F.M. and M.B., validation: L.L.P., B.O.P. and V.V., writing-original draft preparation: D.C., I.V., V.D. and E.H., writing-review and editing: D.C., I.V. and S.S., visualization: E.H. and V.D., supervision: S.S., D.F.M., M.B. and R.C., project administration: L.L.P. All authors have read and agreed to the published version of the manuscript.

Funding: This research received no external funding.

Institutional Review Board Statement: Not applicable.

Informed Consent Statement: Not applicable.

Data Availability Statement: Not applicable.

Acknowledgments: Authors would like to thank Ioana Mureșanu, Emanuel Ștefanescu, Diana Grad, Irina Benedek, Diana Lascu, Iulia Dobra, and Nicoleta Jemna for contributions to this review.

Conflicts of Interest: M.B. was a principal investigator in the Cerebrolysin in Patients with Acute Ischemic Stroke in Asia (CASTA) trial and has previously received honoraria as a speaker and consultant for EVER Neuro Pharma, the manufacturer of Cerebrolysin. D.F.M. was principal investigator in the Cerebrolysin REGistry Study in Stroke (CREGS 2), CARS I, and CAPTAIN I clinical studies funded by EVER Neuro Pharma, as well as the principal investigator in the CAPTAIN II and CAPTAIN rTMS trials, funded academically as part of doctoral studies by the Foundation for the Study of Nanoneuroscience and Neuroregeneration.

\section{References}

1. Chugh, C. Acute Ischemic Stroke: Management Approach. Indian J. Crit. Care Med. Peer-Rev. Off. Publ. Indian Soc. Crit. Care Med. 2019, 23, S140-S146. [CrossRef]

2. Warburton, E.; Alawneh, J.A.; Clatworthy, P.L.; Morris, R.S. Stroke Management. BMJ Clin. Evid. 2011, 6, 201.

3. Phipps, M.S.; Cronin, C.A. Management of Acute Ischemic Stroke. BMJ 2020, 368, 16983. [CrossRef] [PubMed]

4. Muresanu, D.F.; Heiss, W.-D.; Hoemberg, V.; Bajenaru, O.; Popescu, C.D.; Vester, J.C.; Rahlfs, V.W.; Doppler, E.; Meier, D.; Moessler, H.; et al. Cerebrolysin and Recovery After Stroke (CARS) A randomized, placebo-controlled, double-blind, multicenter trial. Stroke 2016, 47, 151-159. [CrossRef] [PubMed]

5. Muresanu, D.F.; Buzoianu, A.; Florian, S.I.; von Wild, T. Towards a Roadmap in Brain Protection and Recovery. J. Cell. Mol. Med. 2012, 16, 2861-2871. [CrossRef] [PubMed]

6. Teng, H.; Li, C.; Zhang, Y.; Lu, M.; Chopp, M.; Zhang, Z.G.; Melcher-Mourgas, M.; Fleckenstein, B. Therapeutic effect of Cerebrolysin on reducing impaired cerebral endothelial cell permeability. Neuroreport 2021, 32, 359-366. [CrossRef] [PubMed]

7. Carey, L.; Walsh, A.; Adikari, A.; Goodin, P.; Alahakoon, D.; De Silva, D.; Ong, K.L.; Nilsson, M.; Boyd, L. Finding the Intersection of Neuroplasticity, Stroke Recovery, and Learning: Scope and Contributions to Stroke Rehabilitation. Neural Plast. 2019, $2019,5232374$. [CrossRef]

8. Muresanu, D.F. Management of Acute Stroke: Neuroprotection. In Stroke; Bornstein, N.M., Ed.; Karger: Basel, Switzerland, 2009; pp. 128-136, ISBN 978-3-8055-9099-0.

9. Muresanu, D.F.; Strilciuc, S.; Stan, A. Current Drug Treatment of Acute Ischemic Stroke: Challenges and Opportunities. CNS Drugs 2019, 33, 841-847. [CrossRef]

10. Muresanu, D.F. Neuroprotection and neuroplasticity-A holistic approach and future perspectives. J. Neurol. Sci. 2007, 257, 38-43. [CrossRef]

11. Muresanu, D.F. Neuroplasticity and Neurorecovery. In Stroke; Bornstein, N.M., Ed.; Karger: Basel, Switzerland, 2009 ; pp. 37-49. [CrossRef]

12. Puderbaugh, M.; Emmady, P.D. Neuroplasticity. In StatPearls; StatPearls Publishing: Treasure Island, FL, USA, 2021.

13. Brainin, M. Cerebrolysin: A Multi-Target Drug for Recovery after Stroke. Expert Rev. Neurother. 2018, 18, 681-687. [CrossRef]

14. Brunet, A.; Datta, S.R.; Greenberg, M.E. Transcription-Dependent and -Independent Control of Neuronal Survival by the PI3K-Akt Signaling Pathway. Curr. Opin. Neurobiol. 2001, 11, 297-305. [CrossRef] 
15. Hutter-Paier, B.; Grygar, E.; Frühwirth, M.; Temmel, I.; Windisch, M. Further evidence that Cerebrolysin ${ }^{\circledR}$ protects cortical neurons from neurodegeneration in vitro. J. Neural Transm. Suppl. 1998, 53, 363-372. [PubMed]

16. Iseda, T.; Nishio, T.; Kawaguchi, S.; Kawasaki, T.; Wakisaka, S. Spontaneous Regeneration of the Corticospinal Tract after Transection in Young Rats: Collagen Type IV Deposition and Astrocytic Scar in the Lesion Site Are Not the Cause but the Effect of Failure of Regeneration. J. Comp. Neurol. 2003, 464, 343-355. [CrossRef] [PubMed]

17. Masliah, E.; Díez-Tejedor, E. The Pharmacology of Neurotrophic Treatment with Cerebrolysin: Brain Protection and Repair to Counteract Pathologies of Acute and Chronic Neurological Disorders. Drugs Today 2012, 48, 3-24. [CrossRef]

18. Wu, H.-Y.; Yuen, E.Y.; Lu, Y.-F.; Matsushita, M.; Matsui, H.; Yan, Z.; Tomizawa, K. Regulation of N-Methyl-D-Aspartate Receptors by Calpain in Cortical Neurons. J. Biol. Chem. 2005, 280, 21588-21593. [CrossRef]

19. Kotter, M.R.; Setzu, A.; Sim, F.J.; Van Rooijen, N.; Franklin, R.J.M. Macrophage Depletion Impairs Oligodendrocyte Remyelination Following Lysolecithin-Induced Demyelination. Glia 2001, 35, 204-212. [CrossRef]

20. Windhagen, A.; Newcombe, J.; Dangond, F.; Strand, C.; Woodroofe, M.N.; Cuzner, M.L.; Hafler, D.A. Expression of Costimulatory Molecules B7-1 (CD80), B7-2 (CD86), and Interleukin 12 Cytokine in Multiple Sclerosis Lesions. J. Exp. Med. 1995, 182, 1985-1996. [CrossRef]

21. Hardingham, G.E.; Arnold, F.J.; Bading, H. A calcium microdomain near NMDA receptors: On switch for ERK-dependent synapse-tonucleus communication. Nat. Neurosci. 2001, 4, 565-566. [CrossRef]

22. Paul, S.; Candelario-Jalil, E. Emerging Neuroprotective Strategies for the Treatment of Ischemic Stroke: An Overview of Clinical and Preclinical Studies. Exp. Neurol. 2021, 335, 113518. [CrossRef]

23. Kandel, E. Principles of Neural Science, 5th ed.; Appleton and Lange; McGraw Hill: New York, NY, USA, 2006; ISBN 978-0-07-139011-8.

24. Auriel, E.; Bornstein, N.M. Neuroprotection in acute ischemic stroke-current status. J. Cell. Mol. Med. 2010, 14, $2200-2202$. [CrossRef]

25. Chen, H.; Tung, Y.-C.; Li, B.; Iqbal, K.; Grundke-Iqbal, I. Trophic Factors Counteract Elevated FGF-2-Induced Inhibition of Adult Neurogenesis. Neurobiol. Aging 2007, 28, 1148-1162. [CrossRef]

26. Zhang, C.; Chopp, M.; Cui, Y.; Wang, L.; Zhang, R.; Zhang, L.; Lu, M.; Szalad, A.; Doppler, E.; Hitzl, M.; et al. Cerebrolysin enhances neurogenesis in the ischemic brain and improves functional outcome after stroke. J. Neurosci. Res. 2010, 88, 3275-3281. [CrossRef]

27. Wronski, R.; Tompa, P.; Hutter-Paier, B.; Crailsheim, K.; Friedrich, P.; Windisch, M. Inhibitory effect of a brain derived peptide preparation on the intracellular Calcium Ca++- dependent protease, calpain. J. Neural Transm. 2000, 107, 145-157. [CrossRef] [PubMed]

28. Sugita, Y.; Kondo, T.; Kanazawa, A.; Itou, T.; Mizuno, Y. Protective effect of FPF 1070 (Cerebrolysin) on delayed neuronal death in the gerbil-detection of hydroxyl radicals with salicylic acid. No Shinkei = Brain Nerve 1993, 45, 325-331. [PubMed]

29. Álvarez, X.A.; Lombardi, V.R.M.; Fernández-Novoa, L.; García, M.; Sampedro, C.; Cagiao, A.; Cacabelos, R.; Windisch, M. Cerebrolysin reduces microglial activation in vivo and in vitro: A potential mechanism of neuroprotection. J. Neural Transm. 2000, 59, 281-292.

30. Hutter-Paier, B.; Grygar, E.; Windisch, M. Death of cultured telencephalon neurons induced by glutamate is reduced by the peptide derivate Cerebrolysin. J. Neural Transm. 1996, 47, 267-273.

31. Rockenstein, E.; Adame, A.; Mante, M.; Moessler, H.; Windisch, M.; Masliah, E. The neuroprotective effects of Cerebrolysin trade mark in a transgenic model of Alzheimer's disease are associated with improved behavioral performance. J. Neural Transm. 2003, 110, 1313-1327. [CrossRef]

32. Rockenstein, E.; Mante, M.; Adame, A.; Crews, L.; Moessler, H.; Masliah, E. Effects of Cerebrolysin on neurogenesis in an APP transgenic model of Alzheimer's disease. Acta Neuropathol. 2007, 113, 265-275. [CrossRef]

33. Jin, Y.; Barnett, A.; Zhang, Y.; Yu, X.; Luo, Y. Poststroke sonic hedgehog agonist treatment improves functional recovery by enhancing neurogenesis and angiogenesis. Stroke 2017, 48, 1636-1645. [CrossRef]

34. Zhang, L.; Chopp, M.; Meier, D.H.; Winter, S.; Wang, L.; Szalad, A.; Lu, M.; Wei, M.; Cui, Y.; Zhang, Z.G. Sonic hedgehog signaling pathway mediates cerebrolysin-improved neurological function after stroke. Stroke 2013, 44, 1965-1972. [CrossRef]

35. Tran, L.; Alvarez, X.A.; Le, H.-A.; Nguyen, D.-A.; Le, T.; Nguyen, N.; Nguyen, T.; Nguyen, T.; Vo, T.; Tran, T.; et al. Clinical Efficacy of Cerebrolysin and Cerebrolysin plus Nootropics in the Treatment of Patients with Acute Ischemic Stroke in Vietnam. CNS Neurol. Disord.-Drug Targets 2021, 20, 281-292. [CrossRef] [PubMed]

36. Chang, W.H.; Lee, J.; Shin, Y.-I.; Ko, M.-H.; Kim, D.Y.; Sohn, M.K.; Kim, J.; Kim, Y.-H. Cerebrolysin Combined with Rehabilitation Enhances Motor Recovery and Prevents Neural Network Degeneration in Ischemic Stroke Patients with Severe Motor Deficits. J. Pers. Med. 2021, 11, 545. [CrossRef] [PubMed]

37. Chang, W.H.; Park, C.; Kim, D.Y.; Shin, Y.-I.; Ko, M.-H.; Lee, A.; Jang, S.Y.; Kim, Y.-H. Cerebrolysin Combined with Rehabilitation Promotes Motor Recovery in Patients with Severe Motor Impairment after Stroke. BMC Neurol. 2016, 16, 31. [CrossRef] [PubMed]

38. Xue, L.-X.; Zhang, T.; Zhao, Y.-W.; Geng, Z.; Chen, J.-J.; Chen, H. Efficacy and Safety Comparison of DL-3-n-Butylphthalide and Cerebrolysin: Effects on Neurological and Behavioral Outcomes in Acute Ischemic Stroke. Exp. Ther. Med. 2016, 11, 2015-2020. [CrossRef]

39. Guekht, A.; Heiss, D.; Gusev, E.; Vester, J.; Doppler, E.; Muresanu, D. Cerebrolysin and Recovery after Stroke (CARS 2): A Randomized, Placebo-Controlled, Double-Blind, Multicenter Clinical Study. J. Neurol. Sci. 2015, 357, e103. [CrossRef] 
40. Rezaei, Y.; Amiri-Nikpour, M.R.; Nazarbaghi, S.; Ahmadi-Salmasi, B.; Mokari, T.; Tahmtan, O. Cerebrolysin Effects on Neurological Outcomes and Cerebral Blood Flow in Acute Ischemic Stroke. Neuropsychiatr. Dis. Treat. 2014, 10, 2299. [CrossRef]

41. Lang, W.; Stadler, C.H.; Poljakovic, Z.; Fleet, D. A Prospective, Randomized, Placebo-Controlled, Double-Blind Trial about Safety and Efficacy of Combined Treatment with Alteplase (Rt-PA) and Cerebrolysin in Acute Ischaemic Hemispheric Stroke. Int. J. Stroke 2013, 8, 95-104. [CrossRef]

42. Stan, A.; Birle, C.; Blesneag, A.; Iancu, M. Cerebrolysin and Early Neurorehabilitation in Patients with Acute Ischemic Stroke: A Prospective, Randomized, Placebo-Controlled Clinical Study. J. Med. Life 2017, 10, 216-222.

43. Heiss, W.-D.; Brainin, M.; Bornstein, N.M.; Tuomilehto, J.; Hong, Z. Cerebrolysin in Patients With Acute Ischemic Stroke in Asia: Results of a Double-Blind, Placebo-Controlled Randomized Trial. Stroke 2012, 43, 630-636. [CrossRef]

44. Bornstein, N.M.; Guekht, A.; Vester, J.; Heiss, W.-D.; Gusev, E.; Hömberg, V.; Rahlfs, V.W.; Bajenaru, O.; Popescu, B.O.; Muresanu, D. Safety and Efficacy of Cerebrolysin in Early Post-Stroke Recovery: A Meta-Analysis of Nine Randomized Clinical Trials. Neurol. Sci. Off. J. Ital. Neurol. Soc. Ital. Soc. Clin. Neurophysiol. 2018, 39, 629-640. [CrossRef]

45. Guekht, A.; Vester, J.; Heiss, W.-D.; Gusev, E.; Hoemberg, V.; Rahlfs, V.W.; Bajenaru, O.; Popescu, B.O.; Doppler, E.; Winter, S.; et al. Safety and Efficacy of Cerebrolysin in Motor Function Recovery after Stroke: A Meta-Analysis of the CARS Trials. Neurol. Sci. 2017, 38, 1761-1769. [CrossRef] [PubMed]

46. Strilciuc, S.; Vécsei, L.; Boering, D.; Pražnikar, A.; Kaut, O.; Riederer, P.; Battistin, L. Safety of Cerebrolysin for Neurorecovery after Acute Ischemic Stroke: A Systematic Review and Meta-Analysis of Twelve Randomized-Controlled Trials. Pharmaceuticals 2021, 14, 1297. [CrossRef]

47. Ziganshina, L.E.; Abakumova, T.; Hoyle, C.H. Cerebrolysin for Acute Ischaemic Stroke. Cochrane Database Syst. Rev. 2020, 7, CD007026. [CrossRef] [PubMed]

48. Gartlehner, G. Criteria for Distinguishing Effectiveness From Efficacy Trials in Systematic Reviews; Technical Reviews; Agency for Healthcare Research and Quality: Rockville, MD, USA, 2006.

49. Martinez, R.M. Efficacy of Cerebrolysin in the Reduction of Spasticity during Stroke Rehabilitation. J. Med. Life 2017, 10, 161-166. [PubMed]

50. Kim, J.Y.; Kim, H.J.; Choi, H.S.; Park, S.Y.; Kim, D.Y. Effects of Cerebrolysin ${ }^{\circledR}$ in Patients With Minimally Conscious State After Stroke: An Observational Retrospective Clinical Study. Front. Neurol. 2019, 10, 803. [CrossRef] [PubMed]

51. Katan, M.; Luft, A. Global Burden of Stroke. Semin. Neurol. 2018, 38, 208-211. [CrossRef]

52. Rochmah, T.N.; Rahmawati, I.T.; Dahlui, M.; Budiarto, W.; Bilqis, N. Economic Burden of Stroke Disease: A Systematic Review. Int. J. Environ. Res. Public. Health 2021, 18, 7552. [CrossRef]

53. Hay, J.W.; Smeeding, J.; Carroll, N.V.; Drummond, M.; Garrison, L.P.; Mansley, E.C.; Mullins, C.D.; Mycka, J.M.; Seal, B.; Shi, L. Good Research Practices for Measuring Drug Costs in Cost Effectiveness Analyses: Issues and Recommendations: The ISPOR Drug Cost Task Force Report-Part I. Value Health 2010, 13, 3-7. [CrossRef]

54. Mauskopf, J.A.; Sullivan, S.D.; Annemans, L.; Caro, J.; Mullins, C.D.; Nuijten, M.; Orlewska, E.; Watkins, J.; Trueman, P. Principles of Good Practice for Budget Impact Analysis: Report of the ISPOR Task Force on Good Research Practices-Budget Impact Analysis. Value Health 2007, 10, 336-347. [CrossRef]

55. Kulikov, A.; Abdrashitova, G. Cost-Effectiveness Analysis of Cerebrolysin In The Treatment of Patients With Acute Ischemic Stroke Moderate and Severe Degrees of Severity In The Russian Federation. Value Health 2015, 18, A705. [CrossRef]

56. Kulikov, A.; Abdrashitova, G. Budget Impact Analysis of Cerebrolysin In The Treatment of Acute Ischemic Stroke of Moderate and Severe Degrees of Severity In The Russian Federation. Value Health 2015, 18, A699. [CrossRef]

57. Walter, E.; Bauer, M.; Ressl, S. Cost-Effectiveness Of Combined Treatment With Alteplase (Rt-Pa) And Cerebrolysin In Acute Ischemic Hemispheric Stroke In Austria. Value Health 2015, 18, A390. [CrossRef]

58. European Observatory of Health Systems and Policies Austria HiT. 2018. Available online: https://www.euro.who.int/en/aboutus/partners/observatory-old/publications/health-system-reviews-hits/full-list-of-country-hits/austria-hit-2018 (accessed on 12 January 2022).

59. Russian Federation Russian Federation: Health System Review. 2011. Available online: https:/ / eurohealthobservatory.who.int/ publications/i/russian-federation-health-system-review-2011 (accessed on 12 January 2022).

60. Teasell, R.; Hussein, N.; Mirkowski, M.; Vanderlaan, D.; Saikaley, M.; Longval, M.; Iruthayarajah, J. Stroke Rehabilitation Clinician Handbook; Heart and Stroke Foundation: London, ON, Canada, 2020.

61. Platz, T.; Fheodoroff, K.; Mehrholz, J. S3 Guideline Rehabilitation Therapy for Arm Paresis after Stroke of the DGNR Long Version; Springer: Greifswald, Germany, 2020.

62. Beghi, E.; Binder, H.; Birle, C.; Bornstein, N.; Diserens, K.; Groppa, S.; Homberg, V.; Lisnic, V.; Pugliatti, M.; Randall, G.; et al European Academy of Neurology and European Federation of Neurorehabilitation Societies guideline on pharmacological support in early motor rehabilitation after acute ischaemic stroke. Eur. J. Neurol. 2021, 28, 2831-2845. [CrossRef] [PubMed]

63. Powers, W.J.; Rabinstein, A.A.; Ackerson, T.; Adeoye, O.M.; Bambakidis, N.C.; Becker, K.; Biller, J.; Brown, M.; Demaerschalk, B.M.; Hoh, B.; et al. Guidelines for the Early Management of Patients With Acute Ischemic Stroke: 2019 Update to the 2018 Guidelines for the Early Management of Acute Ischemic Stroke: A Guideline for Healthcare Professionals From the American Heart Association/American Stroke Association. Stroke 2019, 50, e344-e418. [CrossRef] [PubMed] 
64. Winstein, C.J.; Stein, J.; Arena, R.; Bates, B.; Cherney, L.R.; Cramer, S.C.; Deruyter, F.; Eng, J.J.; Fisher, B.; Harvey, R.L.; et al. Guidelines for Adult Stroke Rehabilitation and Recovery: A Guideline for Healthcare Professionals From the American Heart Association/American Stroke Association. Stroke 2016, 47, e98-e169. [CrossRef]

65. Nagappan, P.G.; Chen, H.; Wang, D.-Y. Neuroregeneration and Plasticity: A Review of the Physiological Mechanisms for Achieving Functional Recovery Postinjury. Mil. Med. Res. 2020, 7, 30. [CrossRef] 